\title{
Wireless Tissue Palpation for Intraoperative Detection of Lumps in the Soft Tissue
}

\author{
Marco Beccani, Student Member, IEEE, Christian Di Natali, Student Member, IEEE, \\ Levin J. Sliker, Student Member, IEEE, Jonathan A. Schoen, Mark E. Rentschler, Member, IEEE, \\ and Pietro Valdastri*, Member, IEEE
}

\begin{abstract}
In an open surgery, identification of precise margins for curative tissue resection is performed by manual palpation. This is not the case for minimally invasive and robotic procedures, where tactile feedback is either distorted or not available. In this paper, we introduce the concept of intraoperative wireless tissue palpation. The wireless palpation probe (WPP) is a cylindrical device $(15 \mathrm{~mm}$ in diameter, $60 \mathrm{~mm}$ in length) that can be deployed through a trocar incision and directly controlled by the surgeon to create a volumetric stiffness distribution map of the region of interest. This map can then be used to guide the tissue resection to minimize healthy tissue loss. The wireless operation prevents the need for a dedicated port and reduces the chance of instrument clashing in the operating field. The WPP is able to measure in real time the indentation pressure with a sensitivity of $34 \mathrm{~Pa}$, the indentation depth with an accuracy of $0.68 \mathrm{~mm}$, and the probe position with a maximum error of $11.3 \mathrm{~mm}$ in a tridimensional workspace. The WPP was assessed on the benchtop in detecting the local stiffness of two different silicone tissue simulators (elastic modulus ranging from 45 to $220 \mathrm{kPa}$ ), showing a maximum relative error below $5 \%$. Then, in vivo trials were aimed to identify an agar-gel lump injected into a porcine liver and to assess the device usability within the frame of a laparoscopic procedure. The stiffness map created intraoperatively by the WPP was compared with a map generated ex vivo by a standard uniaxial material tester, showing less than $8 \%$ local stiffness error at the site of the lump.
\end{abstract}

Index Terms-Force feedback, minimally invasive surgery (MIS), soft tissue identification, surgical robotics, tissue palpation, tumor localization.

\section{INTRODUCTION}

M INIMALLY invasive surgery (MIS) drastically reduces patient trauma and recovery time when compared to an

Manuscript received May 14, 2013; revised August 1, 2013 and June 22, 2013; accepted August 15, 2013. Date of publication August 21, 2013; date of current version January 16, 2014. This work was supported by the National Science Foundation under Grant CNS-1239355 and by the National Center for Advancing Translational Sciences under Grant UL1-TR000445-06. M. Beccani and C. Di Natali equally contributed to this work. Asterisk indicates corresponding author.

M. Beccani and C. Di Natali are with the Science and Technology of Robotics in Medicine Laboratory, Department of Mechanical Engineering, Vanderbilt University, Nashville, TN 37235-1592 USA (e-mail: marco.beccani@ vanderbilt.edu; christian.di.natali@vanderbilt.edu).

L. J. Sliker and M. E. Rentschler are with the Department of Mechanical Engineering, University of Colorado, Boulder, CO 80309 USA (e-mail: levin.sliker@colorado.edu; mark.rentschler@colorado.edu).

J. A. Schoen is with the Department of Surgery at the University of Colorado Hospital, Aurora, CO 80045 USA (e-mail: jonathan.schoen@ucdenver.edu).

${ }^{*} \mathrm{P}$. Valdastri is with the Science and Technology of Robotics in Medicine Laboratory, Department of Mechanical Engineering, Vanderbilt University, Nashville, TN 37235-1592 USA (e-mail: p.valdastri@vanderbilt.edu).

Color versions of one or more of the figures in this paper are available online at http://ieeexplore.ieee.org.

Digital Object Identifier 10.1109/TBME.2013.2279337 open surgery. More than two million MIS procedures are performed annually in the United States alone [1]. Patient benefits, however, come with a price for surgeons in terms of constrained maneuverability of laparoscopic instruments and restricted field of view through the endoscopic camera. The force and tactile cues are commonly used in an open surgery to identify tumor margins and vessels buried in the soft tissue. In MIS, any haptic cue is severely compromised by the use of laparoscopic instruments due to friction against the surgical port (i.e., trocar) and the fulcrum effect at the insertion point [2]. These shortcomings are amplified in the robotic surgery, where the surgeon is physically removed from the bedside and haptic feedback is completely absent in the more than 2000 Intuitive Surgical da Vinci platforms installed worldwide [3].

Tissue palpation is essential to effectively explore nonvisible tissue and organ features, to identify buried structures (e.g., nerves or blood vessels) that must be avoided during the surgical procedure, and to identify precise margins for curative tumor resections [4]. Achieving negative surgical margins is particularly relevant during partial nephrectomies [5] and hepatic surgeries [6], in order to minimize accidental damage to healthy tissue and to prevent organ failure, that would result in the urgent need for a transplant. Registration with preoperative imaging - a standard practice for image-guided surgery [7] —is not a viable option for the soft tissues [4], [8]. Therefore, surgeons currently rely on an intraoperative ultrasonography (IOUS) for the evaluation of vascular anatomy, identification of known and occult lesions, and operative planning [9]. Recent studies confirm the utility of IOUS also in robotic procedures [5], [6], [10], [11], even if several open issues still remain unaddressed. In particular, IOUS can only provide a vertical slice of tissue density, while a stiffness distribution map would better serve the need of tumor margin identification.

Restoring haptic sensations in MIS and robotic MIS has been an active research topic for more than two decades [12], [13], with one of the first systems used in a human dating back to 1994 [14]. A relevant number of MIS instruments with force and/or tactile sensors have been developed to acquire in vivo data for tissue modeling and simulation [15]-[18], to improve the outcomes of the surgical procedure - preventing excessive forces from being applied to the tissues [2], [19]-[22], or to create stiffness distribution map by palpation [4], [8], [23]-[27].

However, MIS palpation instruments developed to date all present a rigid shaft and require a dedicated port. This increases the chance of tool clashing in the operating field and often requires an assistant to operate the palpation probe. A wireless 


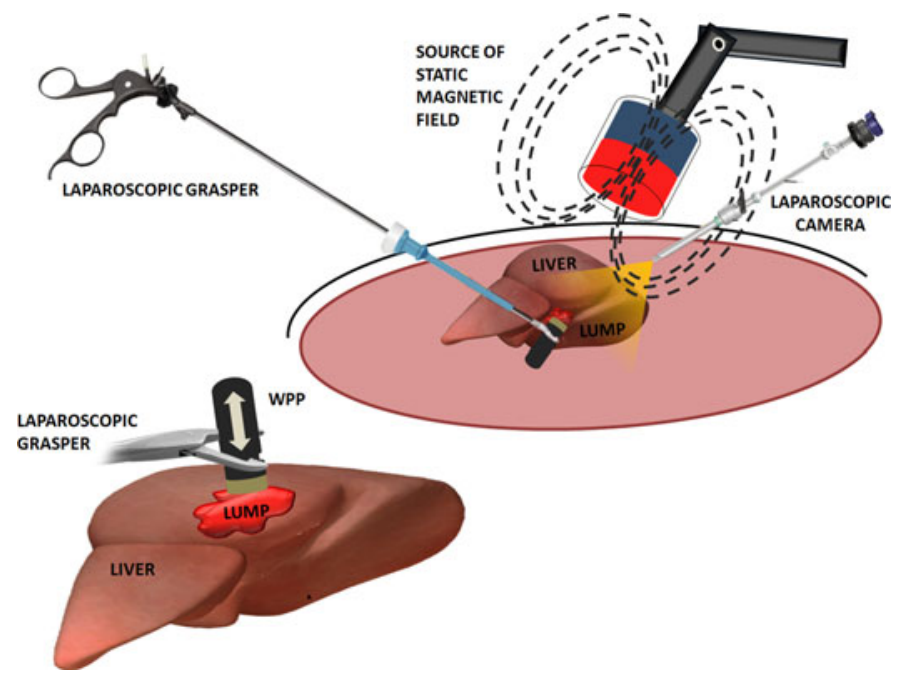

Fig. 1. Principle of operation for wireless tissue palpation using a WPP.

device for uniaxial indentation of soft tissues was preliminary reported by the authors in [28]. Magnetic fields were proposed to indent the tissue and to reposition the probe while scanning the organ surface. This approach proved to be limited in terms of both safety and reliable positioning, due to the rapid variation of the magnetic field strength with distance. Having a wireless device to be directly manipulated by the surgeon would dramatically improve maneuverability, autonomy, and precision, as illustrated by the soft-tethered IOUS probes presented in [5] and [11].

In this paper, we introduce for the first time an intraoperative wireless palpation probe (WPP)—schematically represented in Fig. 1-that can be deployed through a trocar incision and directly controlled by the surgeon to create a stiffness distribution map. Such a map can then be used to localize tumor margins during the soft tissue surgery, thus improving intraoperative diagnostic and interventional decisions. The wireless operation prevents the need for a dedicated port and reduces the chance of instrument clashing in the operating field.

\section{MAterials}

\section{A. Principle of Operation}

For indentation depths that are less than $10 \%$ of the organ thickness, it is possible to assume the tissue as linear elastic [4]. A volumetric stiffness map can then be created by estimating the local tissue stiffness $E(\mathbf{r})$ through the measurement of the indentation depth $\delta(\mathbf{r})$ and the tissue reaction pressure $P(\mathbf{r})$ at different positions $\mathbf{r}$ on the organ surface

$$
E(\mathbf{r}) \simeq \frac{P(\mathbf{r})}{\delta(\mathbf{r})} .
$$

Referring to Fig. 2, we can define $(x, y, z)$ as the global Cartesian coordinate system, $\left(x^{\prime}, y^{\prime}, z^{\prime}\right)$ as the reference frame at the external source of the static magnetic field, and $\left(x_{w}, y_{w}, z_{w}\right)$ as the coordinate system at the WPP. The origin $O$ of $(x, y, z)$ is coincident with the origin $O^{\prime}$ of $\left(x^{\prime}, y^{\prime}, z^{\prime}\right)$, while $z_{w}$ is aligned with the main axis of the device. We can assume the position

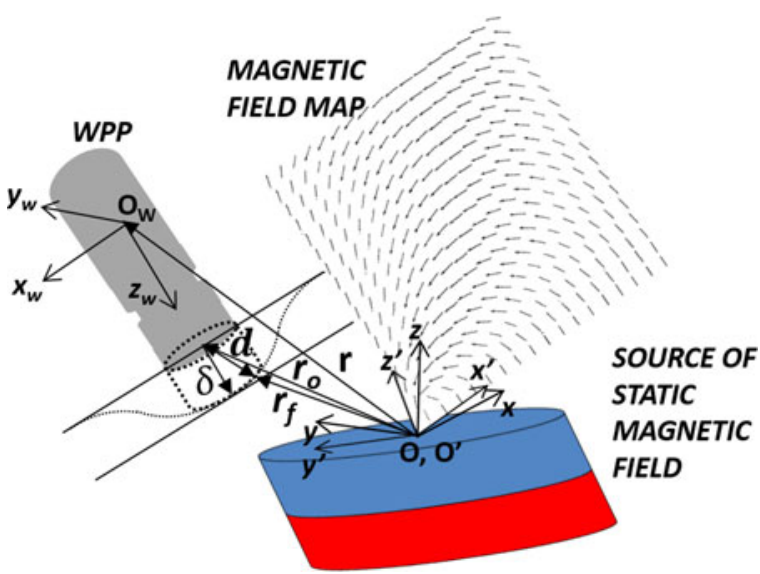

Fig. 2. WPP during indentation and the external source of magnetic field with a slice of the magnetic field map. Vectors $\mathbf{r}_{0}$ and $\mathbf{r}_{f}$ represent the WPP position $\mathbf{r}$ at the beginning and at the end of the indentation. They are represented at the interface of WPP and tissue, rather than at $O_{w}$, for a better understanding of their physical meaning.

vector $\mathbf{r}$ to identify the origin of $\left(x_{w}, y_{w}, z_{w}\right)$-noted as $O_{w}-$ with respect to the global coordinate system $(x, y, z)$. When the WPP is manipulated by the surgeon to palpate a tissue, its motion $\mathbf{d}$ is not constrained along $z_{w}$. Therefore, the following equation must be used to estimate the indentation depth $\delta(\mathbf{r})$ :

$$
\delta(\mathbf{r})=\mathbf{d} \cdot \mathbf{z}_{w 0}=\left(\mathbf{r}_{f}-\mathbf{r}_{0}\right) \cdot \mathbf{z}_{w 0}
$$

where $\mathbf{r}_{0}$ and $\mathbf{r}_{f}$ are the WPP positions at the beginning and at the end of the indentation, respectively, while $\mathbf{z}_{w 0}$ is the unit vector along $z_{w}$ at the beginning of the indentation. In this approach, the beginning of the indentation is identified as the instant when the reading of the tissue reaction pressure $P(\mathbf{r})$ becomes significant. The end of each indentation is identified as the instant when $\delta(\mathbf{r})$ reaches the maximum value.

In this paper, the tissue reaction pressure is acquired by a barometric pressure sensor embedded in a silicone rubber at the probing surface of the WPP. This design is inspired by [29] and further details are provided in Section II-B. A threshold value $P_{\mathrm{th}}$, independent from $\mathbf{r}$, is defined by calibration and takes into account both bias and noise of the pressure sensor. A single indentation starts as $P(\mathbf{r})>P_{\text {th }}$.

Real-time localization of the WPP serves two purposes. First, the position where indentation is taking place must be recorded in three degrees of freedom $(\mathrm{DoF})$ in order to reconstruct the stiffness map. In this case, we assume the position $\mathbf{r}$ of each indentation to be coincident with WPP position as the indentation begins (i.e., $\mathbf{r}_{0}$ ). A second goal for WPP tracking is to derive $\delta(\mathbf{r})$ as in (2). In this case, real-time estimation of $\mathbf{r}$ and rotations of the WPP around $x$ and $y$ are required. Therefore, the WPP position and orientation in five DoF must be available in real time. This is achieved by an on-board localization module, working in synergy with an external source of the static magnetic field, as represented in Fig. 1. The on-board module consists of three orthogonally mounted magnetic field sensors and a triaxial accelerometer (technical details are provided in Section II-B). The accelerometer-used here as an inclinometer-provides WPP rotations around $x$ and $y$. The WPP position vector $\mathbf{r}$ is derived 
from the magnetic field sensor readings, as suggested in [30]. In particular, the magnetic field vector $\mathbf{B}_{w}$ is measured at the WPP and rotated according to

$$
\mathbf{B}=R^{\prime T} R^{w} R^{\prime} \mathbf{B}_{w}
$$

where $R^{w}$ is the rotational matrix of the WPP reference frame with respect to the global Cartesian coordinate system, while $R^{\prime}$ is the rotational matrix of the reference frame at the external source of the static magnetic field with respect to the global Cartesian coordinate system. The matrix $R^{w}$ is obtained in real time from the readings acquired by the inclinometer integrated in the WPP, while $R^{\prime}$ is derived from the data acquired by an inclinometer mounted on the external source of the static magnetic field. Then, a search within a precalculated bidimensional magnetic field map is performed to find the WPP position $\mathbf{r}$ that would match with the actual magnetic field vector $\mathbf{B}$. The magnetic map associates each point $\mathbf{r}$ within the workspaceexpressed in cylindrical coordinates $\left(r_{\rho}, r_{z}\right)$-to the related magnetic field intensity $\mathbf{B}$-also expressed in cylindrical coordinates $\left(B_{\rho}, B_{z}\right)$-with a spatial resolution of $0.2 \mathrm{~mm}$. The third cylindrical coordinate $r_{\theta}$ can be calculated from the values of $B_{x}$ and $B_{y}$ by applying the following equation:

$$
r_{\theta}=\arctan \left(\frac{B_{y}}{B_{x}}\right) \text {. }
$$

The effective localization workspace is a cylinder with a diameter of $35 \mathrm{~cm}$ and a length of $35 \mathrm{~cm}$, centered on the static magnetic field source. The 5-DoF WPP coordinates derived by the algorithm are referenced to a Cartesian frame at the center of the workspace.

\section{B. WPP Development}

Both the methods used to measure the indentation pressure and the solution for WPP localization are designed for wireless operation and can be implemented within a miniature device. The WPP prototype, represented in Fig. 3, has a cylindrical shape ( $15 \mathrm{~mm}$ in diameter, $60 \mathrm{~mm}$ in length, $9.5 \mathrm{~g}$ total mass) with a grasping site close to the pressure sensor head. The cylindrical plastic shell—fabricated by rapid prototyping (OBJET 30, Objet Geometries Ltd, Billerica, MA, USA) — hosts a pressure sensing head, a localization module with a dedicated signal conditioning stage, a power regulation unit, a rechargeable battery, and a wireless microcontroller.

The pressure sensing head-based on the design reported in [29] — consists of a barometric pressure sensor (MPL115A1, Freescale Semiconductors, Austin, TX, USA) embedded in a $2.2 \mathrm{~mm}$-thick silicone rubber layer (VytaFlex 20, Smooth On, Easton, PA, USA). The bare barometric pressure sensor has a sensitivity of $0.5 \mathrm{kPa}$ and a sensing range of $65 \mathrm{kPa}$ for the atmospheric pressure.

The localization module includes three Hall effect sensors (CYP15A, Chen Yang Technologies GmbH \& Co. KG, Finsing, Germany) and a 16-bit triaxial accelerometer with serial peripheral interface (SPI) (LIS331AL, STMicroelectronics, Geneva, Switzerland). The Hall effect sensors are mounted on three orthogonal sides of a cubic structure, as represented in Fig. 3.

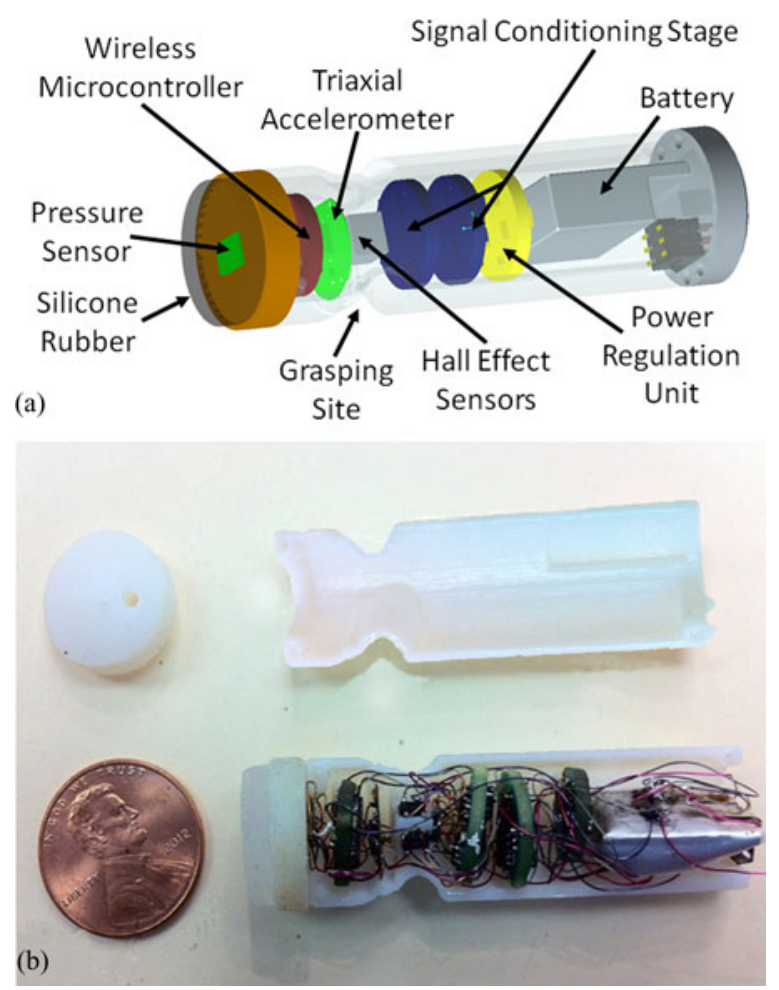

Fig. 3. (a) Schematic view. (b) Picture of the WPP. The signal conditioning stage, the triaxial accelerometer, the power regulation unit, and the wireless microcontroller are mounted on separate printed circuit board (PCB) with a diameter of $9.9 \mathrm{~mm}$. In particular, the signal conditioning stage was separated into two boards due to PCB area constraints.

Their analog outputs are acquired by a signal conditioning stage. This stage consists of three instrumentation amplifiers (AD623, Analog Devices, Norwood, MA, USA) with a unity gain, three low-pass filters $\left(F_{c}=30 \mathrm{~Hz}\right)$, and three 16-bit analog to digital converters (ADS8320, Texas Instruments, Dallas, TX, USA) with SPI interface. The digitalized magnetic field signal has a sensitivity of $0.6 \mathrm{mT}$.

The power regulation unit embeds a low-dropout voltage regulator (TPS73xx, Texas Instruments, Dallas, TX, USA), an operational amplifier (ADS8617, Analog Device, Norwood, MA, USA) used as a voltage divider to provide the proper power supply to the signal conditioning stage and to monitor the battery level. A $50 \mathrm{mAh}, 3.7 \mathrm{~V}$ rechargeable LiPo battery (Shenzhen Hondark Electronics Co., Ltd., Shenzhen, China, $12 \mathrm{~mm} \times 15 \mathrm{~mm} \times 3 \mathrm{~mm}$ in size) is used as the on-board power supply source.

The data from the barometric pressure sensor, the accelerometer, and the magnetic field sensors are acquired by a wireless microcontroller (CC2530, Texas Instruments, Dallas, TX, USA) through the SPI interface at a clock frequency of $1 \mathrm{Mbit} / \mathrm{s}$. Each dataset is then bounded into a 28-byte payload together with a progressive package indicator, a time stamp, the battery level, and two synchronization start and stop bytes. This payload is transmitted by the wireless microcontroller to an external transceiver over a $2.4-\mathrm{GHz}$ carrier frequency. The external transceiver consists of a mirror wireless microcontroller (CC2530, Texas Instruments, Dallas, TX, USA) connected to 
the Universal Serial Bus (USB) port of a personal computer (PC) through a dedicated module (UM232R, FTDI, Glasgow, U.K.).

While the total time required to acquire a single dataset from all the sensors is $3.7 \mathrm{~ms}$, the wireless data throughput runs at $44.8 \mathrm{kbit} / \mathrm{s}$, resulting in a refresh time of $5 \mathrm{~ms}$ and a sampling rate of $200 \mathrm{~Hz}$. The overhead allows to handle correctly the synchronization with the external transceiver.

In addition to the transceiver and the PC, the external platform includes the source of the static magnetic field used for WPP tracking. The magnetic field is generated by an off-theshelf cylindrical $\mathrm{NdFeB}$ permanent magnet mounted on an articulated three-DoF friction clutch arm (Dectron, Roswell, GA, USA). The selected magnet has N52 axial magnetization, magnetic remanence of $1.48 \mathrm{~T}$, is $50 \mathrm{~mm}$ in diameter and $50 \mathrm{~mm}$ in height, and has a mass of $772 \mathrm{~g}$. These features allow for a localization workspace that extends $15 \mathrm{~cm}$ away from each side of the magnet. A triaxial accelerometer (LIS331AL, STMicroelectronics, Geneva, Switzerland) is mounted on the magnet to measure its inclination and derive its rotation with respect to the global reference frame $(x, y, z)$. Accelerometer data are fed directly to the PC through a secondary USB connection.

As concerns waterproofing of the WPP to operate during the surgery, a layer of paraffin film (Parafilm, Sigma Aldrich, St. Louis, MO, USA) was wrapped around the device. An additional layer of film was secured at the grasping site to enable a safe grip.

\section{Communication Protocol and User Interface}

The communication protocol provides robust operation, realtime data acquisition, and low power consumption. A sleep timer is used to wake up the WPP from a low-power mode every $15 \mathrm{~s}$. When active, the WPP tries to establish a wireless communication with the external transceiver. If this attempt fails, the WPP returns in sleep mode to save power. Once the wireless link is established, the WPP acquires a full dataset of sensor readings, transmits it to the external transceiver, and waits for an acknowledgement. If the acknowledgment is received, the WPP continues to acquire and send data. Otherwise, the WPP retries to transmit the same package. This attempt is repeated for two times, then, the firmware forces the device to get a new dataset and updates the payload. In case of loss of the synchronization, the WPP autoresets itself ready for a new acquisition. This protocol allows for a fail safe operation and prevents the need for a hard reset of the device that would not be possible during surgery.

All the data received by the external transceiver are transmitted to the PC together with the received signal strength indicator (RSSI). The RSSI quantifies the quality of the wireless link. In case of a low RSSI, the user is warned to modify the position of the external transceiver to improve the wireless coupling.

A multithread $\mathrm{C}++$ application running on the $\mathrm{PC}$ unbounds the data and shares them with a parallel application developed in MATLAB (Mathworks, Natick, MA, USA) via TCP-IP communication. Refresh rate for displayed data runs at $30 \mathrm{~Hz}$.
The user interface is conceived to work in two different modalities: 1) creation of the volumetric stiffness map and 2) display of WPP position on the volumetric stiffness map. In the first modality, the surgeon grasps the WPP and creates the map by palpating the region of interest. In this case, the user interface displays in real time the $x, y, z$ coordinates of the WPP, a plot of the indentation pressure, and the numeric value of the indentation depth in case the indentation pressure has exceeded $P_{\text {th }}$. Visual indicators are provided to warn the user if the WPP is outside the localization workspace. Once the region of interest has been palpated with the desired spatial resolution, a command is provided by the user through the keyboard to create the volumetric stiffness map. Once the map is available, the user interface switches to the second modality, overlaying the real-time position of the WPP in a 3-D space centered on the map. Under the assumption that the region palpated does not undergo substantial movements, the surgeon can manipulate the WPP as a cursor to identify the margins of a stiffer region buried underneath the tissue.

\section{WPP Characterization}

Before assessing the overall functionality of the proposed device, the single components were tested and characterized on the benchtop. In particular, the first step was to verify the localization unit algorithm to evaluate the WPP workspace, localization error, and any influence of surgical tool in the localization unit performance. Then, a load cell was adopted to calibrate the pressure sensor response. Finally, the WPP electronic performance was tested on bench to assess the battery lifetime and the wireless link reliability.

1) Localization: The device was mounted on the end effector of a six DoF industrial robot (RV6SDL, Mitsubishi Corp., Tokyo, Japan) which was used as a reference position system given its encoder feedback. Assuming the $(x, y, z)$ global reference system centered on the external magnet and having $z$ aligned with the main axis of the magnet, we characterized the localization on a grid of 3 by 3 points equally spaced by $50 \mathrm{~mm}$ along $x$ - and $y$-directions at three different $z$ coordinates (i.e., 80,110 , and $140 \mathrm{~mm}$ ). For each position, localization data were acquired from the robot encoders and the WPP algorithm. Onboard localization was repeated for each point with a disposable laparoscopic grasper (EndoGrasp $5 \mathrm{~mm}$, Covidien, Mansfield, MA, USA) closing its jaws at the grasping site. Then, the indentation depth error was estimated at each point of the grid by moving the robot end effector $3 \mathrm{~mm}$ along $z$ in open air, thus emulating palpation. The average absolute errors were equal to $4.7 \mathrm{~mm}( \pm 4.5 \mathrm{~mm})$ for $x, 4.1 \mathrm{~mm}( \pm 5.8 \mathrm{~mm})$ for $y$, and $4.5 \mathrm{~mm}$ $( \pm 2.2 \mathrm{~mm}$ ) for $z$. The laparoscopic grasper increased the localization error to $9.8 \mathrm{~mm}( \pm 5.1 \mathrm{~mm})$ for $x, 11.3 \mathrm{~mm}( \pm 6.6 \mathrm{~mm})$ for $y$, and $10.6 \mathrm{~mm}( \pm 4.6 \mathrm{~mm})$ for $z$. However, we observed that the contribution of the laparoscopic grasper does not vary substantially within the workspace, thus, it can be assumed as a constant offset that factors out when reconstructing the stiffness map. The indentation depth average absolute error resulted in $0.68 \mathrm{~mm}( \pm 0.44 \mathrm{~mm})$. 


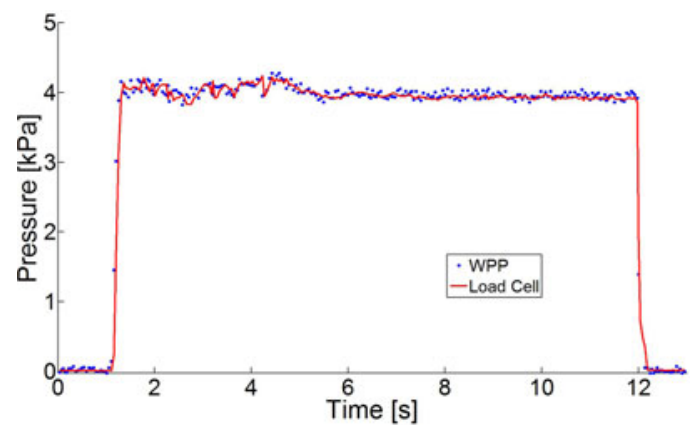

Fig. 4. Step response of the WPP pushing against a reference load cell.

2) Pressure Sensing Head: To calibrate and characterize the pressure sensing head response, a 6-DoF load cell (NANO17, ATI Industrial Automation, Apex, NC, USA, resolution 1/160 N) was adopted as the force reference system [26]. The WPP was mounted as in the previous experiment, while the load cell was fixed on the benchtop. A 1-mm step motion pushing the WPP against the load cell was imposed. Speed of motion was $65 \mathrm{~mm} / \mathrm{s}$. After about $9 \mathrm{~s}$, the same step was imposed in the opposite direction, releasing the load. From the experimental results-represented in Fig. 4-we can conclude that the silicone layer embedding the barometric pressure sensor does not introduce any relevant delay in the sensor response. An additional set of trials was performed by pushing the WPP against the load cell at a lower speed (i.e., $3.12 \mathrm{~mm} / \mathrm{s}$ ), until the saturation of the barometric pressure sensor occurred. This test was repeated five times. The pressure sensing head showed a sensitivity of $P_{s}=34 \mathrm{~Pa}$ (i.e., considering the probing area, this is equivalent to $5 \mathrm{~g}$ or $0.049 \mathrm{~N}$ ), while saturation occurred at $P_{\mathrm{SAT}}=5 \mathrm{kPa}$ (i.e., considering the probing area, this is equivalent to $730 \mathrm{~g}$ or $7.16 \mathrm{~N}$ ). In light of a recent study [27] that reports tissue damage to the liver for a force exceeding $6 \mathrm{~N}$-exerted by a probing area of the same size of the WPP-we can conclude that the pressure sensing range is adequate for this exploratory investigation. The threshold value $P_{\text {th }}$ was therefore assumed as $P_{\mathrm{th}}=P_{\mathrm{bias}}+2 P_{s}$, where $P_{\mathrm{bias}}$ is the output value for the sensor when unloaded. This value for $P_{\mathrm{th}}$ allowed us to reliably identify the start of an indentation.

3) Electronics: As regards power consumption, a single 5ms loop of data acquisition and wireless transmission drains an average of $33.3 \mathrm{~mA}$ with a peak of $41.6 \mathrm{~mA}$. This translates in a battery lifetime of about $90 \mathrm{~min}$ when the WPP is in the active mode. The average current consumption drops down to $3 \mathrm{~mA}$ when the WPP is in low-power mode.

The data synchronization between the WPP and the external transceiver was tested in open air to estimate the robustness of the protocol. The firmware was run for 36 consecutive hours without failures and was then stopped. The results included a package loss below $2 \%$ and an average RSSI of $-13.5 \mathrm{dBm}$ at a distance of $2 \mathrm{~m}$ between the WPP and the external transreceiver. Complete loss of communication occurs as the RSSI drops below $-88 \mathrm{dBm}$.

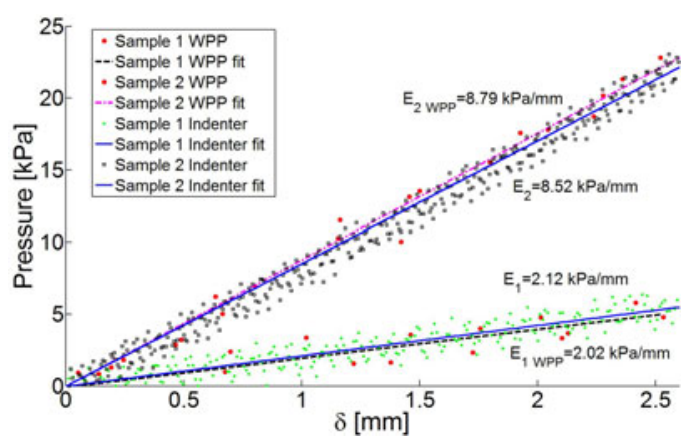

Fig. 5. Experimental data acquired by standard and wireless indentation for two different silicone samples. For this trial, relative errors in local stiffness identification were equal to $4.72 \%$ for sample 1 and $3.17 \%$ for sample 2 .

\section{WPP ASSESSMENT}

Experimental validation of the proposed platform consisted in two different trials. First, the effectiveness of the probe in identifying the local stiffness of a tissue simulator was assessed. Then, in vivo trials were aimed to identify agar-gel lumps injected into a porcine liver and to assess the device usability within the frame of an MIS procedure.

\section{A. Assessment of Local Stiffness Identification}

To estimate the ability of the WPP in detecting different local stiffnesses, two different synthetic tissue samples were fabricated combining two different ratios of liquid plastic and hardener (PVC Regular Liquid Plastic - Hardener, MF Manufacturing, Fort Worth, TX, USA-Sample 1: 1 to 10 ratio, resulting in an elastic modulus of $220 \mathrm{kPa}$; Sample 2: 1 to 2 ratio, resulting in an elastic module of $45 \mathrm{kPa}$ ). The samples were $30 \mathrm{~mm}$ thick with a lateral side of $75 \mathrm{~mm}$. A traditional indenter was obtained mounting a 6-DoF load cell (MINI 45, ATI Industrial Automation, Apex, NC, USA, resolution $1 / 16 \mathrm{~N}$ ) at the end effector of the robotic manipulator used previously. A cylindrical probe with the same contact surface as the WPP was mounted on the distal side of the load cell to indent the sample. Then, the cylindrical probe was replaced with the WPP and the indentation was repeated acquiring both the indentation pressure and depth from the wireless device. Five loading-unloading trials reaching an indentation depth of $2.6 \mathrm{~mm}$ were performed for each tissue sample and each method at a constant speed of $0.75 \mathrm{~mm} / \mathrm{s}$. The local stiffnesses measured with the traditional indenter were equal to $\mathrm{E}_{1}=2.12 \mathrm{kPa} / \mathrm{mm}, \mathrm{E}_{2}=8.52 \mathrm{kPa} / \mathrm{mm}$, while the results obtained with the WPP were $\mathrm{E}_{1 \mathrm{WPP}}=2.02 \mathrm{kPa} / \mathrm{mm}$, $\mathrm{E}_{2 \mathrm{WPP}}=8.79 \mathrm{kPa} / \mathrm{mm}$. Experimental plots obtained from a single loading are represented in Fig. 5. Overall, the WPP was effective in detecting the local stiffness of different samples with an average relative error equal to $4.7 \%$ for sample 1 and $3 \%$ for sample 2.

\section{B. In Vivo Validation}

The feasibility of wireless tissue palpation was then assessed in vivo on an anesthetized porcine model. The primary measure of interest was to acquire a volumetric stiffness map of a 


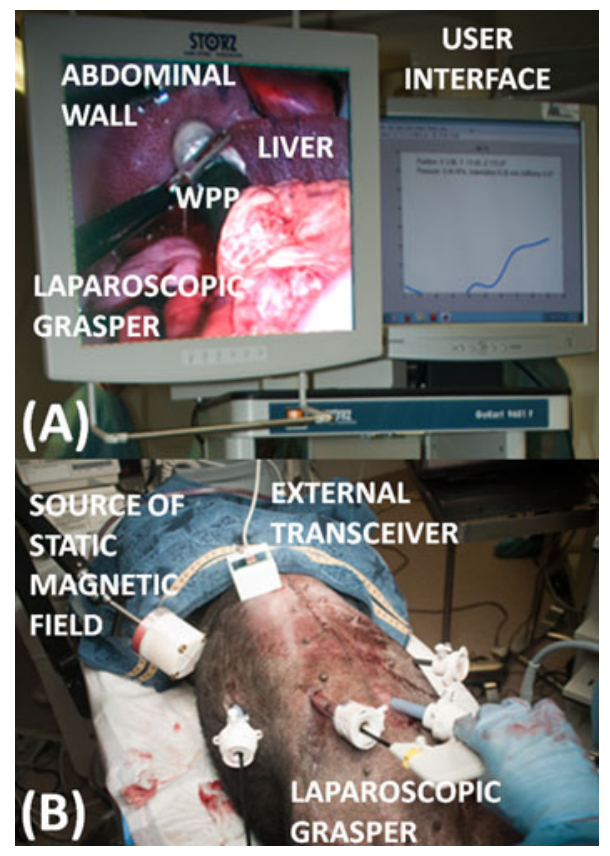

Fig. 6. Picture of the surgical setup during the in vivo trial. (a) Snapshot of the laparoscopic camera view and the user interface during the creation of the volumetric stiffness map. (b) Picture of the surgical field.

segment of the liver where agar-gel was injected to simulate a hepatic tumor. The map acquired in vivo by wireless palpation was then compared with a stiffness map obtained post-mortem within $12 \mathrm{~h}$ after the procedure using a standard uniaxial material tester. Secondary measures of interest were the time to scan a liver segment by wireless palpation, WPP usability, instrument clashing, and operator workload. Reliability of the wireless link was also assessed.

The porcine surgery was performed at the University of Colorado Anschutz Medical Campus under IACUC protocol 87912(04)1D. A 57-kg female standard pig was used for this study. After intravenous sedation, a laparotomy was performed to access the liver. Similarly to the methods suggested in [8], 6 cc of Sigma Gelrite Gellan Gum (agar) was prepared in a 30:1 ratio of water to agar by weight, boiled, and injected into the right lateral segment of the liver, to approximately the midthickness of the organ. The midline incision was then sutured, and minimally invasive access was gained by one 5-mm (5 Versaport Plus, Covidien, Norwalk, CT, USA) and three 12-mm trocars (512 Versaport Plus, Covidien, Norwalk, CT, USA). The WPP was introduced in the abdominal cavity through one of the 12-mm trocar incisions before the placement of the port. The external source of the magnetic field and the external transceiver were placed in the close vicinities of the right side of the animal, as represented in Fig. 6(b). The surgeon used a standard laparoscopic grasper to operate the WPP under endoscopic guidance (see Fig. 7). A lateral screen showed in real-time WPP position in three DoF, indentation pressure, and indentation depth [see Fig. 6(a)].

Once the right segment of the liver was identified, the surgeon palpated the organ in different positions, always targeting at least $3 \mathrm{~mm}$ as the indentation depth. To prevent localization

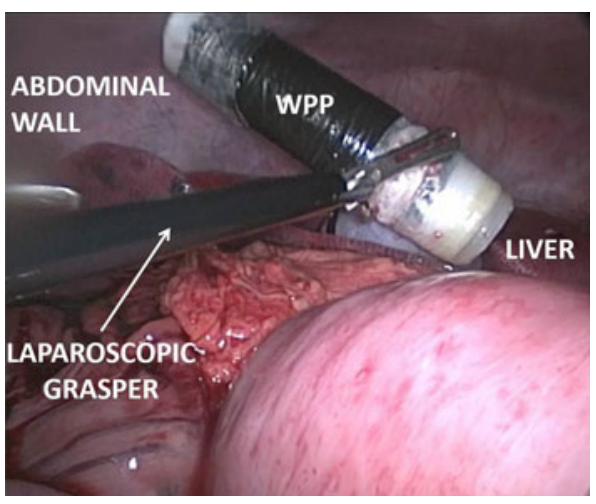

Fig. 7. Laparoscopic view of the WPP operated by the surgeon through a laparoscopic grasper during in vivo trials.

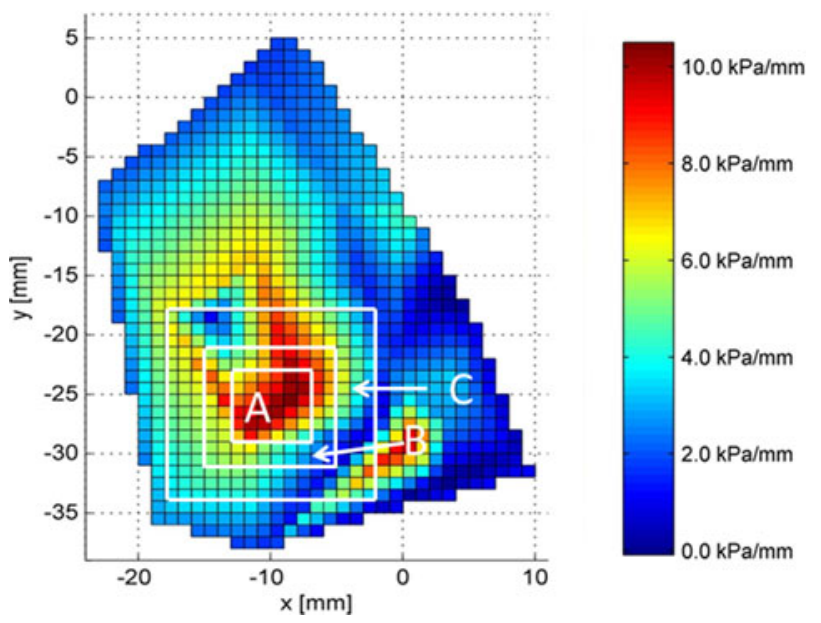

Fig. 8. Local stiffness map acquired in vivo for a $6 \mathrm{cc}$ agar-gel lump injected into the liver. Since the surface of the liver was almost flat in the palpated region, a bidimensional projection of the map is shown. The local stiffness values inside areas A, B, and C were compared with the ex vivo map represented in Fig. 9.

artifacts, the surgeon verified that the liver was not moving during palpation and that adequate support was provided by the rib cage and the surrounding organs. Tissue stiffness was acquired on a total of 30 different points on the liver surface. This required about $5 \mathrm{~min}$. The local stiffness map, represented in Fig. 8, was then generated by the algorithm and displayed on the lateral screen, overlaying the current position of the WPP.

Immediately after euthanization, the liver was harvested from the animal for ex vivo palpation tests using a standard uniaxial material testing system (MTS) (Insight 2 Electromechanical Testing System, MTS System Corporation, Eden Prairie, MN, USA) to create a comparable stiffness map. The liver was placed in $0.9 \%$ phosphate buffered saline (PBS) solution immediately after excision and refrigerated until the ex vivo tests were performed. The liver was warmed to room temperature prior to testing. The organ was placed on a platform, marked with 28 pins and photographed from the top (see Fig. 9). The liver was indented with a cylindrical indenter probe (2-mm diameter) beside each pin location - to avoid palpating tissue that had been pricked by the pin. The test was performed following a standard tissue compressive property measurement method [31]. The tissue was hydrated throughout the tests by spraying PBS on the 


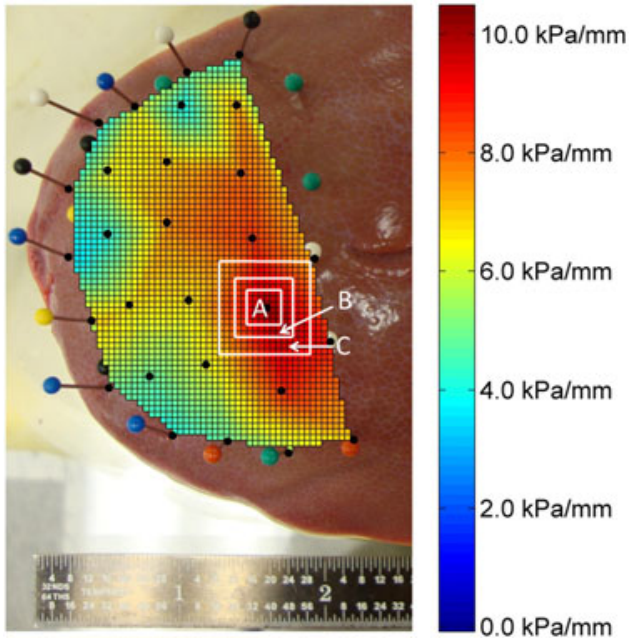

Fig. 9. Stiffness map obtained with a standard uniaxial MTS, overlayed on the right lateral segment of the explanted porcine liver. The local stiffness values inside areas $\mathrm{A}, \mathrm{B}$, and $\mathrm{C}$ were compared with the in vivo map represented in Fig. 8.

surface prior to each indentation. The testing room conditions were $23.5^{\circ} \mathrm{C}$ and $22 \%$ relative humidity. A 2-N load cell (PN LCCA-118-75, MTS System Corporation, Eden Prairie, MN, USA) with 1-mN resolution was used to measure the load exerted on the tissue by the indenter during each indentation. The probe was programmed to approach the surface of the tissue at a low speed $(0.1 \mathrm{~mm} / \mathrm{s})$ until a force threshold $(2 \mathrm{mN})$ was reached. At that point, the probe advanced into the tissue at a rate of $1 \mathrm{~mm} / \mathrm{s}$ to a depth of $3 \mathrm{~mm}$ to simulate the in vivo experiment. The force and indentation depth (10 $\mu \mathrm{m}$ resolution) data were collected at $100 \mathrm{~Hz}$ and analyzed with a customized program developed in MATLAB. Following testing, the tissue was resected to verify that the agar did not dilute in the liver.

The force data were divided by the surface area of the cylindrical probe tip to obtain pressure. The local stiffness at each point was determined by computing the slope of a linear regression of the first $0.75 \mathrm{~mm}$ of the pressure-displacement curve. The force at depths larger than $0.75 \mathrm{~mm}$ were found to be too high due to the rigid platform that the liver was resting on and the relatively small liver thickness. This was not an issue in vivo as the liver was pressed against other organs or the rib cage. The stiffness values were assigned to pin locations and overlaid on the photograph of the liver to produce the stiffness map shown in Fig. 9.

The two local stiffness maps were then compared with MAT$\mathrm{LAB}$ (grid area is equal to $1 \mathrm{~mm}^{2}$ for both the maps). In particular, the maximum measured stiffness resulted in $10.0 \mathrm{kPa} / \mathrm{mm}$ with the MTS machine versus $10.8 \mathrm{kPa} / \mathrm{mm}$ with the WPP, corresponding to a $8 \%$ relative error. Then, the average pseudo stiffness of the three different areas A $\left(36 \mathrm{~mm}^{2}\right), \mathrm{B}\left(64 \mathrm{~mm}^{2}\right)$, and $\mathrm{C}\left(156 \mathrm{~mm}^{2}\right)$ centered on the maximum point were compared. Area $\mathrm{A}$ is a square sided $6 \mathrm{~mm}$, area B is the frame with outer dimension $10 \mathrm{~mm}$, and inner dimension $6 \mathrm{~mm}$, while the area $\mathrm{C}$ is the frame with outer dimension $16 \mathrm{~mm}$ and inner dimension $10 \mathrm{~mm}$. The three areas are shown in both the Figs. 8 and 9. The average stiffness was equal to $E_{A_{\mathrm{M} \mathrm{TS}}}=9.64 \mathrm{kPa} / \mathrm{mm}$ and $E_{A_{\mathrm{WPP}}}=8.87 \mathrm{kPa} / \mathrm{mm}$ (average relative error $7.96 \%$ ), $E_{B_{\mathrm{MTS}}}=9.20 \mathrm{kPa} / \mathrm{mm}$ and $E_{B_{\mathrm{WPP}}}=6.58 \mathrm{kPa} / \mathrm{mm}$ (average relative error $28.5 \%$ ) and $E_{C_{\mathrm{M} \mathrm{T}}}=8.64 \mathrm{kPa} / \mathrm{mm}$ and $E_{C_{\mathrm{WPP}}}=$ $4.82 \mathrm{kPa} / \mathrm{mm}$ (average relative error $44.2 \%$ ). The tissue stiffness slightly increased after euthanization and throughout the MTS testing due to the preservation and dehydration. However, the stiffness at the injection site remained constant to the in vivo conditions because the gel properties did not vary after explantation. This can help explain why the relative error increases with the distance from the maximum point which is nearby the injection site.

As concerns the qualitative measures of interest, no instrument clashing was reported. However, the length of the WPP limited the range of motion whenever the target of palpation was too close to the ribcage. The operator workload was minimal, since the surgeon was able to use a standard laparoscopic instrument to operate the WPP. Relevant learning occurred just at the beginning of the procedure, when the surgeon had to understand how strong to grasp the WPP to prevent slippage. This required about $20 \mathrm{~min}$. After that, the surgeon was able to operate the WPP without losing the grip. The wireless link was always reliable, resulting in an average RSSI of $-33.4 \mathrm{dBm}$ with losses between $4.8 \%$ and $6.2 \%$ of the total packages. Battery operation was effective for the entire procedure.

It is worth mentioning that the surgeon noted that a tether tied to the WPP would help in the retrieval at the end of the procedure. A wired connection may also provide power to the WPP instead of the battery, thus allowing for a reduction in size. On the other hand, a tether may limit WPP motion and get trapped in between instruments.

\section{CONCLUSIONS AND FUTURE WORK}

This paper introduces for the first time the concept of wireless tissue palpation to localize tumor margins intraoperatively by creating a stiffness distribution map in real time. The proposed wireless device is manipulated directly by the surgeon through a standard grasper, thus improving autonomy, precision, and maneuverability. Wireless operation effectively prevents instrument clashing and removes from the need of a dedicated access port. Preliminary in vivo results showed the feasibility of acquiring a stiffness map during a minimally invasive procedure. In the future, this map can be used to guide liver resection without sacrificing excess normal tissue and preventing postoperative organ failure.

While the indentation pressure is acquired by a sensor mounted on-board, the position and the indentation depth measurements rely on an external source of the static magnetic field. This imposes a constraint on the workspace, since the magnetic field strength drops exponentially with distance. With the proposed platform, the workspace is a cylinder with a diameter of $35 \mathrm{~cm}$ and a length of $35 \mathrm{~cm}$, centered on the source of the static magnetic field. Considering that the abdominal wall thickness for severely obese patients (Body Mass Index $\leq 40 \mathrm{~kg} / \mathrm{m}^{2}$ ) is usually below $4 \mathrm{~cm}$ [32], the proposed platform is easily applicable to the vast majority of patients undergoing the abdominal surgery. Nevertheless, if a larger workspace is required, either 
the source of the magnetic field or the on-board magnetic field sensors can be adapted to meet the desired requirements.

As previously mentioned, motion of the organ during the creation of the map or poor background support for the tissue may result in localization artifacts. If this occurs, the surgeon needs to restart the acquisition of stiffness values. This issue is common for intraoperative palpation and can be addressed with appropriate surgical planning.

Next steps will consist of improving localization accuracy, [25] reducing the size of the WPP - to achieve a better maneuverability - and in demonstrating how the WPP can be used to assist liver resection in a series of in vivo trials. Blinded studies will be performed, where the operator is not aware of the location/number/stiffness of the buried lumps. In these studies, the effectiveness of the WPP approach will be compared with other forms of intraoperative palpation. Also, additional bench trials will be performed to quantify the efficacy of tumor identification with respect to size, depth, and relative stiffness of embedded lumps, following a protocol similar to [4] and performing CT scans of the region of interest as a benchmark for localization. The repeatability of the results will be quantified through statistical analysis. Nonlinear stiffness modeling will be considered for the detection of deeper tumors. A triaxial force sensor [33]-[36] may be used in the probing head of the WPP instead of a uniaxial pressure sensor. This would allow for studying more complex interactions with the tissue and to improve lump margin detection. Another relevant future step will be the optimization of the user interface. This will include a study on the most effective way to convey the acquired information to the surgeon, along the lines of the results reported in [25].

\section{REFERENCES}

[1] J. Fuller, W. Scott, B. Ashar, and J. Corrado. (2003). "Laparoscopic trocar injuries: A report from a U.S. Food and Drug Administration (FDA) Center for Devices and Radiological Health (CDRH) Systematic Technology Assessment of Medical Products (STAMP) committee," [Online]. Available: www.fda.gov/medicaldevices/safety/alertsandnotices/ ucm197339.htm

[2] P. Puangmali, H. Liu, L. Seneviratne, P. Dasgupta, and K. Althoefer, "Miniature 3-axis distal force sensor for minimally invasive surgical palpation," IEEE/ASME Trans. Mechatronics, vol. 17, no. 4, pp. 646-656, Aug. 2012.

[3] Intuitive Surgical website. (2013). [Online]. Available: www. intuitivesurgical.com

[4] H. Liu, J. Li, X. Song, L. Seneviratne, and K. Althoefer, "Rolling indentation probe for tissue abnormality identification during minimally invasive surgery," IEEE Trans. Robot., vol. 27, no. 3, pp. 450-460, Jun. 2011.

[5] B. F. Kaczmarek, S. Sukumar, F. Petros, Q. D. Trinh, N. Mander, R. Che, M. Menon, and C. Rogers, "Robotic ultrasound probe for tumor identification in robotic partial nephrectomy: Initial series and outcomes," Int. J. Urol., vol. 20, no. 2, pp. 172-176, 2013.

[6] C. M. Schneider, P. D. Peng, R. H. Taylor, G. W. Dachs, C. J. Hasser, S. P. DiMaio, and M. A. Choti, "Robot-assisted laparoscopic ultrasonography for hepatic surgery," Surgery, vol. 151, no. 5, pp. 756-762, 2012.

[7] S. D. Herrell, R. L. Galloway, and L. M. Su, "Image-guided robotic surgery: Update on research and potential applications in urologic surgery," Curr. Opin. Urol., vol. 22, no. 1, pp. 47-54, 2012.

[8] G. McCreery, A. Trejos, M. Naish, R. Patel, and R. Malthaner, "Feasibility of locating tumours in lung via kinaesthetic feedback.," Int. J. Med. Robot. Comput. Assist. Surg., vol. 4, no. 1, pp. 58-68, 2008.

[9] E. C. Lai, C. N. Tang, J. P. Ha, D. K. Tsui, and M. K. Li, "The evolving influence of laparoscopy and laparoscopic ultrasonography on patients with hepatocellular carcinoma," Amer. J. Surg., vol. 196, no. 5, pp. 736740, 2008.

[10] R. Yakoubi, R. Autorino, H. Laydner, J. Guillotreau, M. A. White, S. Hillyer, G. Spana, R. Khanna, W. Isaac, G. P. Haber, R. J. Stein, and J. H. Kaouk, "Initial laboratory experience with a novel ultrasound probe for standard and single-port robotic kidney surgery: Increasing console surgeon autonomy and minimizing instrument clashing," Int. J. Med. Robot., vol. 8, no. 2, pp. 201-205, 2012.

[11] L. Fratt, "Ultrasound opens new doors in robotic surgery," Health Imag., vol. 10, no. 5, pp. 12-17, May/Jun. 2012.

[12] P. Dario and M. Bergamasco, "An advanced robot system for automated diagnostic tasks through palpation," IEEE Trans. Biomed. Eng., vol. 35, no. 2, pp. 118-126, Feb. 1988.

[13] R. Howe, W. Peine, D. Kantarinis, and J. Son, "Remote palpation technology," IEEE Eng. Med. Biol. Mag., vol. 14, no. 3, pp. 318-323, May/Jun. 1995.

[14] T. Ohtsuka, A. Furuse, T. Kohno, J. Nakajima, K. Yagyu, and S. Omata, "Application of a new tactile sensor to thoracoscopic surgery: Experimental and clinical study," Ann. Thoracic Surg., vol. 60, no. 3, pp. 610-614, 1995.

[15] M. Ottensmeyer and J. Salisbury, "In vivo data acquisition instrument for solid organ mechanical property measurement," in Proc. Med. Image Comput. Comput.-Assist. Interven., 2001, pp. 975-982.

[16] E. Samur, M. Sedef, C. Basdogan, L. Avtan, and O. Duzgun, "A robotic indenter for minimally invasive measurement and characterization of soft tissue response," Med. Image Anal., vol. 11, no. 4, pp. 361-373, 2007.

[17] J. Rosen, J. Brown, S. De, M. Sinanan, and B. Hannaford, "Biomechanical properties of abdominal organs in vivo and postmortem under compression loads," J. Biomech. Eng., vol. 130, no. 021020, pp. 1-17, 2008.

[18] K. Lister, Z. Gao, and J. Desai, "Development of in vivo constitutive models for liver: Application to surgical simulation," Ann. Biomed. Eng., vol. 39, pp. 1060-1073, 2011.

[19] O. Van der Meijden and M. Schijven, "The value of haptic feedback in conventional and robot-assisted minimal invasive surgery and virtual reality training: A current review," Surg. Endosc., vol. 23, pp. 1180-1190, 2009.

[20] G. Tholey and J. P. Desai, "A compact and modular laparoscopic grasper with tri-directional force measurement capability," ASME J. Med. Devices, vol. 2, no. 3, pp. 031 001-9, 2008.

[21] B. Kübler, U. Seibold, and G. Hirzinger, "Development of actuated and sensor integrated forceps for minimally invasive robotic surgery," Int. J. Med. Robot. Comput. Assist. Surg., vol. 1, no. 3, pp. 96-107, 2005.

[22] M. Stark, T. Benhidjeb, S. Gidaro, and E. Morales, "The future of telesurgery: A universal system with haptic sensation," J. Turkish-German Gynecolog. Assoc., vol. 13, no. 1, pp. 74-76, 2012.

[23] J. Gwilliam, Z. Pezzementi, E. Jantho, A. Okamura, and S. Hsiao, "Human versus robotic tactile sensing: Detecting lumps in soft tissue," in Proc. IEEE Hapt. Symp., 2010, pp. 21-28.

[24] R. E. Goldman, A. Bajo, and N. Simaan, "Algorithms for autonomous exploration and estimation in compliant environments," Robotica, vol. 31, no. 1, pp. 71-87, 2013.

[25] A. Talasaz and R. V. Patel, "Integration of force reflection with tactile sensing for minimally invasive robotics-assisted tumor localization," IEEE Trans. Haptics, vol. 6, no. 2, pp. 217-228, 2013.

[26] M. T. Perri, A. L. Trejos, M. D. Naish, R. V. Patel, and R. A. Malthaner, "Initial evaluation of a tactile/kinesthetic force feedback system for minimally invasive tumor localization," IEEE/ASME Trans. Mechatronics, vol. 15, no. 6, pp. 925-931, Dec. 2010.

[27] M. T. Perri, A. L. Trejos, M. D. Naish, R. V. Patel, and R. A. Malthaner, "New tactile sensing system for minimally invasive surgical tumour localization," Int. J. Med. Robot. Comput. Assist. Surg., vol. 6, no. 2, pp. 211220, 2010.

[28] M. Beccani, C. Di Natali, M. E. Rentschler, and P. Valdastri, "Wireless tissue palpation: Proof of concept for a single degree of freedom," in Proc. IEEE Int. Conf. Robot. Autom., 2013, pp. 703-709.

[29] Takktile by Y. Tenzer, L. Jentoft, I. Daniher, and R. Howe. (2013). [Online]. Available: www.takktile.com

[30] C. Di Natali, M. Beccani, and P. Valdastri, "Real-time pose detection for magnetic medical devices," IEEE Trans. Magn., vol. 49, no. 7, pp. 3524-3527, 2013.

[31] X. Wang, J. A. Schoen, and M. E. Rentschler, "Quantitative comparison of soft tissue compressive viscoelastic model accuracy," J. Mech. Behav. Biomed. Mater, vol. 20, pp. 126-136, 2013.

[32] S. L. Best, R. Bergs, M. Gedeon, J. Paramo, R. Fernandez, J. A. Cadeddu, and D. J. Scott, "Maximizing coupling strength of magnetically anchored 
surgical instruments: How thick can we go?" Surg. Endosc., vol. 25, no. 1, pp. 153-159, 2011.

[33] P. Valdastri, K. Harada, A. Menciassi, L. Beccai, C. Stefanini, M. Fujie, and P. Dario, "Integration of a miniaturised triaxial force sensor in a minimally invasive surgical tool," IEEE Trans. Biomed. Eng., vol. 53, no. 11, pp. 2397-2400, Nov. 2006.

[34] Y. Hu, R. Katragadda, H. Tu, Q. Zheng, Y. Li, and Y. Xu, "Bioinspired 3-D tactile sensor for minimally invasive surgery," J. Microelectromech. Syst., vol. 19, no. 6, pp. 1400-1408, 2010.

[35] J. Peirs, J. Clijnen, D. Reynaerts, H. V. Brussel, P. Herijgers, B. Corteville, and S. Boone, "A micro optical force sensor for force feedback during minimally invasive robotic surgery," Sens. Actuators A, Phys., vol. 115, no. 2, pp. 447-455, 2004.

[36] P. Baki, G. Székely, and G. Kósa, "Design and characterization of a novel, robust, tri-axial force sensor," Sens. Actuators A, Phys., vol. 192, no. 1, pp. 101-110, 2012.

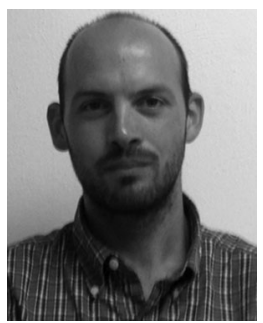

Marco Beccani (S'11) received the Master's degree in electronic engineering from the University of Pisa, Pisa, Italy, in 2010. After spending one year as Research Assistant at the Institute of BioRobotics of Scuola Superiore Sant'Anna, since 2011, he is working toward the $\mathrm{Ph} . \mathrm{D}$. degree in mechanical engineering at Vanderbilt University, Nashville, TN, USA.

$\mathrm{He}$ is a member of the Science and Technology Of Robotics in Medicine Laboratory, and his field of research is miniaturized real-time embedded system design for wireless robotic capsular endoscopy and

robotic surgery.

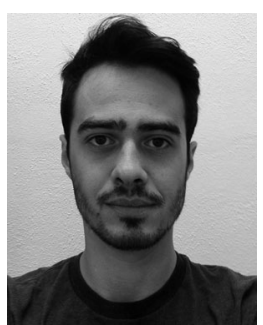

Christian Di Natali (S'10) received the Master's degree (Hons.) in biomedical engineering from the University of Pisa, Pisa, Italy in 2010. Since Fall 2011, he has been working toward the Ph.D. degree in mechanical engineering at Vanderbilt University, Nashville, TN, USA.

In 2011, he joined the Institute of BioRobotics of Scuola Superiore Sant'Anna, as a Research Assistant working on magnetic coupling and teleoperated magnetic navigation. He is a member of the STORM lab, and he is actively involved in the design of advanced magnetic coupling for surgery and endoscopy, controlled mechatronic platforms, and magnetic localization.

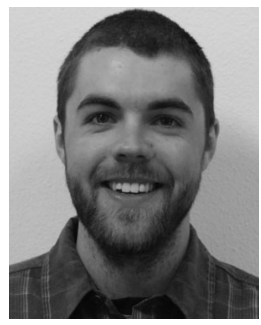

Levin J. Sliker (S'13) received the B.S. and M.S. degrees in mechanical engineering from the University of Colorado, Boulder, CO, USA, in 2010 and 2012, respectively, where he is currently working toward the Ph.D. degree in mechanical engineering, where he is a National Science Foundation (NSF) Graduate Research Fellow.

His research interests include dynamic contact experimentation and modeling, medical robot design, and mechatronics.

Mr. Sliker is a member of the American Society

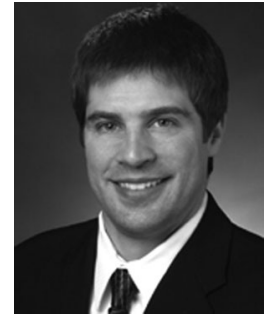

Jonathan A. Schoen received the B.S. degree in biology from Colgate University, Hamilton, NY, USA, in 1993, the M.A. degree in biomedical sciences from Touro College, Dix Hills, NY, USA, in 1994, and the M.D. degree from the Technion Institute of Technology, Haifa, Israel, in 1998. His internship, residency and fellowship were all at the University of Colorado Health Sciences Center, Denver, CO.

He is currently a Board Certified Surgeon specializing in bariatric surgery and advanced minimally invasive surgery, including laparoscopic gastrointestinal surgery and general surgery at the University of Colorado Hospital, Aurora, CO, USA. His research interests include the mechanisms of weight loss after gastric bypass, as well as developing a unique integrated and structured exercise and fitness plan following surgery, to further improve long-term weight loss and health.

Dr. Schoen is an active member of the American Society for Bariatric Surgeons and the Society of American Gastrointestinal Endoscopic Surgeons.

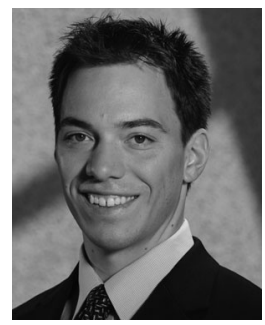

Mark E. Rentschler (M'08) received the B.S. degree in mechanical engineering from the University of Nebraska, Lincoln, NE, USA, the M.S. degree in mechanical engineering from the Massachusetts Institute of Technology, Cambridge, MA, USA, where he was a National Defense Science and Engineering Graduate Fellow, and the Ph.D. degree in biomedical engineering from the University of Nebraska, Lincoln, NE, USA.

$\mathrm{He}$ is currently an Assistant Professor, the CoDirector of Design Center Colorado, and the Director of the Graduate Design Program in Mechanical Engineering at the University of Colorado in Boulder, Boulder, CO, USA. He also holds a secondary appointment in the Department of Surgery at the University of Colorado Anschutz Medical Campus, Aurora, CO, USA, and holds an affiliate position in the Department of Bioengineering at the University of Colorado at Denver, Denver, CO, USA. Previously, he had been a Postdoctoral Researcher in the Division of Vascular Surgery at the University of Nebraska Medical Center, Omaha, NE, USA, and the Senior Engineer and the Director of Operations at Virtual Incision Corporation, Boston, MA, USA. His research interests include medical mechatronics and surgical robotics design, tissue mechanics characterization, and tissue-device interaction modeling.

Dr. Rentschler has performed research at the NASA Goddard Space Flight Center, Greenbelt, MD, USA, and is also a member of the American Society of Mechanical Engineers.

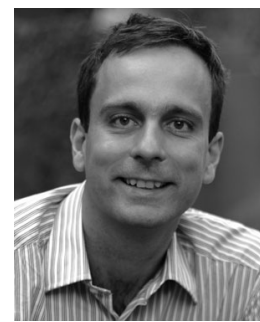

Pietro Valdastri (M'05) received the Master's (Hons.) degree in electronic engineering from the University of Pisa, Pisa, Italy, in 2002, and the Ph.D. degree in biomedical engineering from the Scuola Superiore Sant'Anna (SSSA), Pisa, Italy.

After spending three years as an Assistant Professor at the Institute of BioRobotics of SSSA, since 2011 he has been an Assistant Professor at the Department of Mechanical Engineering at Vanderbilt University, Nashville, TN, USA, where he founded the Science and Technology Of Robotics in Medicine Lab. He is actively involved in robotic endoscopy and robotic surgery, design of magnetic mechanisms, and design of capsule robots for medical applications. 